\title{
Property Patterns for Runtime Monitoring of Web Service Conversations
}

\author{
Jocelyn Simmonds, Marsha Chechik, and Shiva Nejati \\ University of Toronto, Toronto ON M5S3G4, Canada \\ \{jsimmond, chechik, shiva\}ecs.toronto.edu
}

\begin{abstract}
For a system of distributed processes, correctness can be ensured by statically checking whether their composition satisfies the properties of interest. However, web services are distributed processes that dynamically discover properties of other web services. Since the overall system may not be available statically and since each business process is supposed to be relatively simple, we propose to use runtime monitoring of conversations between partners as a means of checking behavioral correctness of the entire web service system. Specifically, we identify a subset of UML 2.0 Sequence Diagrams (SD) as a property specification language. We show how our language can be used to specify the Specification Property System (SPS) [1]. By formalizing this subset using automata, we can check finite execution traces of web services against various complex properties. Finally, we discuss our experience using our language for runtime monitoring of an existing application, and conclude with a description of existing tool support.
\end{abstract}

\section{Introduction}

Web services are collections of components which discover and bind to other components using published interfaces, with support of Service-Oriented Architectures (SOA). The goal of SOA is to increase the flexibility of business interactions. Each web service component can be written in a traditional compiled language such as Java, or in an XML-centric language such as BPEL [2].

Consider, for example, a web-based Loan Application system (LA), distributed as a sample application with the IBM Websphere Integration Developer v6.0.2. Users enter loan application information (name, taxpayer id, loan amount) through a web page, and are eventually informed of the status of their applications. The LA workflow first checks the user's credit score and declines a loan if the user has a bad credit score, i.e., less than 750. If the credit score is good, the workflow then checks the loan amount: loans for $\$ 50,000$ or less are automatically approved; loans for larger amounts are earmarked for manual approval.

The workflow diagram in Fig. 1(a) shows high level steps that are executed in a loan application system. Specifically, this figure shows the BPEL specification of the main LA component together with interaction between its partners. In order to accomplish its goal, the LA system needs to invoke its partners: such as CreditCheck (implemented in Java), rule groups (LoanLimit), or human tasks (Fol lowUpDecl inedApp, CompleteTheLoan and ProcessTheApplication). These partners are shown in Fig. 1(b) and implement the following functions: CheckCredit uses the taxpayer id to retrieve the corresponding credit score; the LoanLimit rule group checks the credit score and the loan amount. The human tasks represent the following application results: declined, approved and manual approval, respectively. 


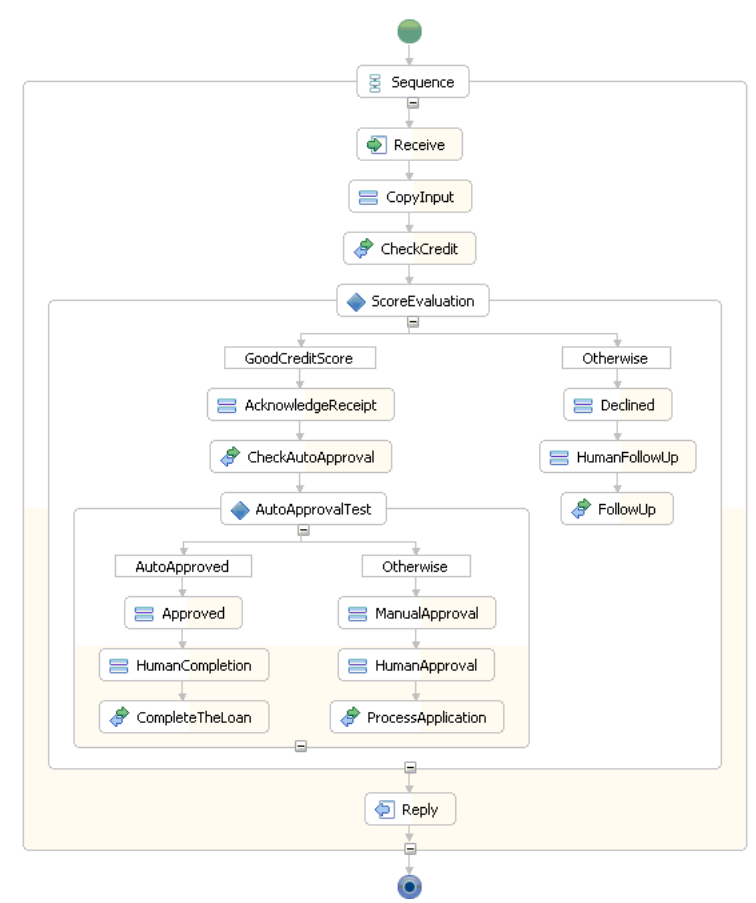

(a)

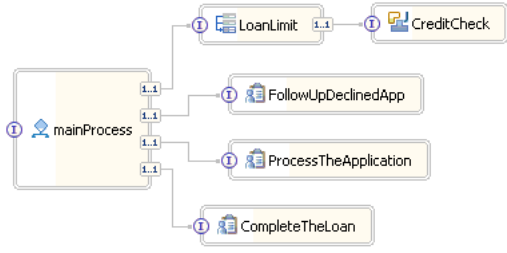

(b)

Fig. 1: LA system: (a) workflow; (b) assembly diagram.

Since the LA system is a composition of several distributed business processes, its correctness depends on correctness of its partners. For example, the system should guarantee that every request is eventually acknowledged and none are lost or blocked indefinitely, or that loans are only given to customers with a good credit score. However, in the provided LA application, the CheckCredit module assigns a credit score at random, without using the customer id, thus preventing the overall system from satisfying this property.

Since each web service is a relatively simple process, analysis can concentrate on the message exchange between partners - their conversations. While static techniques for checking partner composition against properties of interest, such as [3-7], are appealing, they have a number of limitations: the problem is decidable only under certain conditions [8], since the partners communicate via infinite-sized channels, and existing techniques are unable to deal with complex message interactions and heterogeneity of partners.

Instead, we concentrate on the dynamic analysis via runtime monitoring. Unlike the work of [9-11], our approach is to create an industrial-strength (with partnership with the IBM Toronto Lab) monitoring framework that is non-intrusive, supports the dynamic discovery of web services, deals with synchronous and asynchronous communication and partners implemented in different languages, allows for specifying and efficient monitoring of a variety of temporal behaviour, and is usable by practitioners.

Looking for a visual language that allows specification of events, has an explicit emphasis on components, and is able to deal with positive and negative scenarios of interaction as well as global properties, we have, in [12], chosen a subset of UML 2.0 Sequence Diagrams [13] as our specification language. We have shown that this sub- 
\begin{tabular}{|l|l|}
\hline$P_{1}$ & The loan amount must be always greater than zero. \\
\hline$P_{2}$ & The credit score should eventually be checked if the loan amount is greater than zero. \\
\hline$P_{3}$ & A loan cannot be granted if the loan amount is less than or equal to zero. \\
\hline$P_{4}$ & $\begin{array}{c}\text { After checking that the applicant has a good credit score, A loan cannot be granted } \\
\text { if the loan amount is less than or equal to zero. }\end{array}$ \\
\hline$P_{5}$ & No one can get a loan without first going through a credit check. \\
\hline
\end{tabular}

Table 1: Some properties of the LA system.

set is sufficiently expressive for capturing safety (nothing bad can ever happen) and liveness (something good will eventually happen) properties. For example, for the LA system described earlier, possible safety and liveness properties are $P_{1}$ and $P_{2}$, respectively (see Table 1). While liveness properties are not monitorable in general, they can be effectively checked for web services with finitely terminating behaviours. For example, we can check whether the LA process terminates without giving feedback to the customer.

To enable monitoring, [12] formalized the chosen subset of Sequence Diagrams using finite-state automata. These automata are then used in the implementation of our non-intrusive monitoring framework which runs in parallel with the system being monitored, intercepting events from web service conversations. The resulting system enables conformance checking of finite execution traces against their specifications expressed in our subset of Sequence Diagrams.

In [12], we showed that the chosen specification language is capable of capturing simple safety and liveness properties, in particular, invariants, e.g., $P_{1}$ in Table 1, and request-response properties, e.g., $P_{2}$ in Table 1 . However, we need a more expressive language to conveniently specify and verify various system properties that arise in practice, e.g., $P_{4}$ and $P_{5}$ in Table 1 . In this paper, we extend the specification language of [12] by enriching this language with several operators, adopted from UML 2.0 [13] and other scenario-based languages [14]. Examples of these operators include criti$c a l$, ref (which allows to reuse portions of sequence diagrams in other diagrams) and message complementation. Furthermore, in contrast to our previous work [12], we allow arbitrary nested applications of assert and negate operators. We then show that the resulting language can not only be converted into finite-state automata for monitoring, but is also sufficiently expressive to capture a wide variety of frequently used properties, captured and catalogued in the Specification Pattern System (SPS) [1]. This approach also gives basis for tool support to enable usable specification of runtime conversations.

The rest of this paper is organized as follows. We describe syntax of the subset of UML 2.0 sequence diagrams used for expressing properties of webservice conversations in Section 2. Such properties are then converted into monitoring automata using the techniques discussed in Section 3. We then show how our specification language can be used to specify the complete set of temporal logic property patterns in Section 4 . We describe the implementation of the runtime monitoring framework and report on the result of applying our framework to the LA system in Section 5. Finally, we conclude the paper in Section 6 with a summary of the paper, comparison with related work, and an outline of future research directions.

\section{A Language for Specifying Conversations}

We choose a subset of UML 2.0 Sequence Diagrams as our language for specifying web service conversations. Sequence Diagrams [13] is a popular formalism for model- 


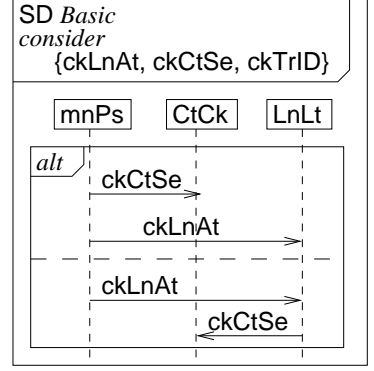

(a)

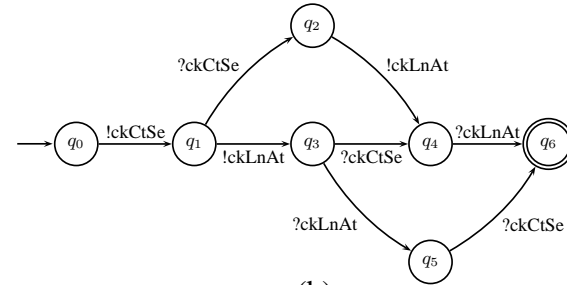

(b)

Fig. 2: (a) An SD describing a scenario of the LA example; and (b) the NFA corresponding to the first argument of the alt operator in Fig 2(a).

ing behavioural scenarios by describing sequences of messages communicated between different objects over time. Sequence Diagrams have two dimensions: vertical, representing time, and horizontal, representing objects. Each object is illustrated by a rectangle with a vertical dashed line, called a lifeline. Lifelines are connected by horizontal arrows denoting messages that are sent from one object to another, synchronously or asynchronously.

An example sequence diagram describing a scenario of the LA system is shown in Fig. 2(a). The diagram contains three objects, mnPs, CtCk, and LnLt. Object $\mathrm{mnP} s$ corresponds to the main workflow of the LA system, and CtCk and LnLt correspond to components CheckCredit and LoanLimit, respectively. The diagram in Fig. 2(a) shows two alternative scenarios: In the first alternative, mnPs first sends a check credit score request, i.e., ckCtSe, to $\mathrm{CtCk}$ and then a check loan amount request, i.e., ckLnAt, to LnLt. In the second alternative, LnLt receives a check loan amount request from mnPs. Since the credit score has not yet been checked, LnLt sends a check credit score request to $\mathrm{CtCk}$.

In UML 2.0, Sequence Diagrams can be augmented by a large number of operators to capture various complex scenarios. We use the operators described below in our property specification language. We refer to our language as $S D$.

Compositional operators: Operators parallel (par), alternatives (alt), strict sequencing (strict seq), and weak sequencing (weak seq) are used to combine two SDs based on standard notions of compositions. The operator loop is used for repeating the scenario described by an SD multiple times, opt - for denoting an optional scenario, equivalent to alt with only one argument. Finally, critical is used to ensure atomicity of the enclosed sequence.

Alphabet changing operators: Operators consider and ignore are used for modifying the communicating alphabet of SDs.

Assertion and negation operators: Operators assert and negate allow users to express mandatory and forbidden system scenarios, respectively.

Interaction use operator: SDs can be shared by reference, using the ref operator. This is a shorthand for copying the contents of the referred SD where the ref operator occurs, and is a new feature in UML 2.0.

To describe system scenarios, we often need to express complementation of individual or a group of messages. Since arbitrary and nested use of the negate operator is inconvenient, we use an operator message complementation, originally introduced in the Property Sequence Charts (PSC) language [14], to negate individual or sets of messages. We denote the complement of a message $m$ by $\neg m$ and define it as the set of all messages that are potentially exchanged between objects of the system except for $m$. 


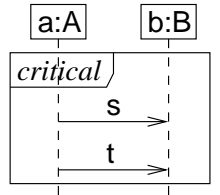

(a)

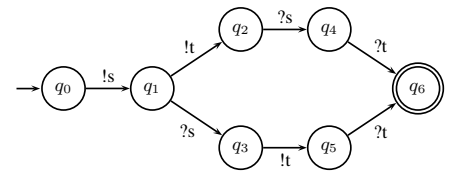

(b)

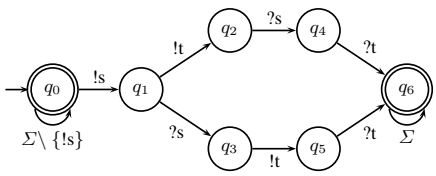

(c)

Fig. 3: (a) A basic SD enclosed by a critical operator; and its corresponding NFAs: (b) before applying critical; (c) after applying critical.

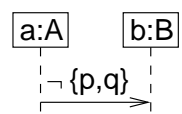

(a)

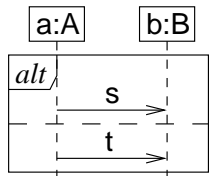

(b)

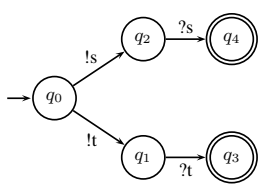

(c)

Fig. 4: (a) An SD with message complementation; (b) the same SD after eliminating the complement operator; and (c) its corresponding NFA.

\section{From SDs to Automata}

We define the formal semantics of SD by translating it into non-deterministic finite automata (NFAs), following the approach of [15]. This translation allows us to not only formalize our language but also to study its expressiveness. Specifically, in [12], we have shown that certain scenarios in SD can be captured by particular forms of NFAs known as Safe and Live automata [16], indicating that SD is capable of expressing safety and liveness properties. In what follows, we briefly review the translation of basic sequence diagrams and the operators described in Section 2 into NFA. We then discuss that the negate and assert operators allow us to express safety and liveness properties, respectively.

Basic sequence diagrams, i.e, diagrams describing a sequence of events without any additional operator, can be translated into NFAs using the procedure in [15]. Consider the scenario in the first argument of the alt operator in Fig 2(a). This basic sequence diagram shows that mnPs first sends event ckCt Se to CtCk and then event ckLnAt to LnLt. We denote the sending of a message $\mathrm{ckCtSe}$ by !ckCt Se and its receiving by ?ckCtSe. Thus, the set of events of the sequence diagram in Fig. 2(a) is $\{$ !ckCtSe, ?ckCtSe, !ckLnAt, ?ckLnAt . Intuitively, lifelines and message arrows in a sequence diagram define a partial order on the set of events of that diagram. Given a basic sequence diagram $S$, an NFA $A_{S}$ is equivalent to $S$ iff $A_{S}$ accepts exactly the set of traces that respect the partial order of $S$. For example, the automaton $A_{S}$ corresponding to the scenario in the first argument of the alt operator in Fig 2(a) is shown in Fig 2(b).

The semantics of the compositional operators can be given in terms of the standard operations defined on NFAs (e.g., see [17]). For example, alt corresponds to the union operator; strict seq corresponds to the sequential composition operator; and loop corresponds to the Kleene star operator.

Operators consider and its dual, ignore, are used to change the set of communicating alphabets of an SD. Both of them receive an SD $S$ and a set of events $E$ as input, but consider adds the elements in $E$ to the set of events of $S$, whereas ignore removes the elements in $E$ from the set of events of $S$. 


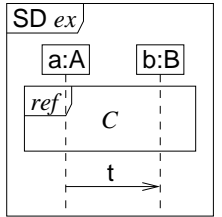

(a)

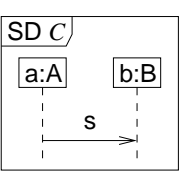

(b)

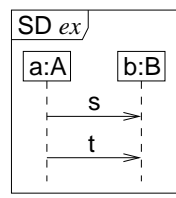

(c)

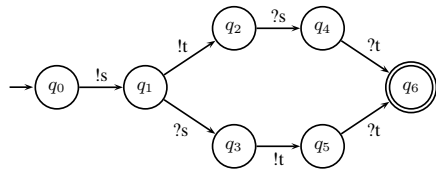

(d)

Fig. 5: (a) An SD with references $\mathrm{SD} C$; (b) $\mathrm{SD} C$; (c) $\mathrm{SD} e x$ after copying the content of $\mathrm{SD} C$; and (d) its corresponding NFA.

We can specify a critical region in a sequence diagram using the critical operator. A critical region means that the traces of the region cannot be interleaved by other messages and thus should be treated atomically. We treat this operator to mean that if the first message of the critical region is observed, then the rest of the behavior must be observed as well.

Let $S$ be an SD enclosed within a critical operator, and let $A_{S}$ be the automaton for $S$. The automaton for critical $S$ is obtained by adding a self-loop at the initial state, $q_{0}$, of $A_{S}$ labelled by $\Sigma \backslash\left\{e \mid q_{0}\right.$ has an outgoing transition on $\left.e\right\}$. This self-loop transition at the initial state allows the automaton to wait for a satisfying run to begin. The initial state also becomes final. For example, Fig. 3(a) shows a sequence diagram with a critical operator, and Fig. 3(c) - its corresponding automaton.

The operator ref is used for sharing portions of SDs between several others. Our treatment of ref is to inline the SD being referenced, applying the necessary translation rules to the result in order to obtain the corresponding NFA, as illustrated in Fig. 5.

To deal with the message complement operator, we note that if $\Sigma$ is the set of messages exchanged in an SD, and $m \in \Sigma$, then $\neg m$ is $\Sigma \backslash\{m\}$. For a set $\{m, n\}$ of messages, $\neg\{m, n\}=\Sigma \backslash\{m, n\}$. For example, let $\Sigma=\{\mathrm{p}, \mathrm{q}, \mathrm{s}, \mathrm{t}\}$. Then, $\neg \mathrm{p}=$ $\{\mathrm{q}, \mathrm{s}, \mathrm{t}\}$ and $\neg\{\mathrm{p}, \mathrm{q}\}=\{\mathrm{s}, \mathrm{t}\}$. Given an SD with a message complement operator $\neg$, we first eliminate this operator by applying an alt operator to the complement of the set of negated messages. Formally, let $S \subseteq \Sigma$ be a set of messages. We replace $\neg S$ by an SD fragment in which operator alt is applied to single messages in $\Sigma \backslash S$. For example, consider the SD in Fig. 4(a) with message, $\neg\{\mathrm{p}, \mathrm{q}\}$, and let $\Sigma=\{\mathrm{s}, \mathrm{t}, \mathrm{p}, \mathrm{q}\}$. This SD is equivalent to that in Fig. 4(b) where $\neg\{$ p, q $\}$ is replaced by an alt fragment in which $s$ and $t$ are two alternative messages. The NFA for the sequence diagram without message complement operators can be generated in a straightforward way following the translation for the alt operator (see Fig. 4 (c)).

Representing safety properties. To describe a safety property, we enclose an SD $S$ within a negate operator to indicate that the scenario represented by $S$ is a forbidden one, and therefore, a safe system should never produce this scenario [16]. For example, the SD in Fig. 6(a) describes the safety property $P_{1}$ in Table 1 . To obtain the automaton for negate $S$, we first derive an NFA $A_{S}$ for SD $S$ and add a self-loop to its initial state to let the automaton guess the beginning of the accepting trace (see Fig. 6(b)). We then determinize and complement $A_{S}$ to obtain an automaton for negate $S$. We have not shown the final automton here because the determinzation step increases the size of $A_{S}$ considerably. The resulting complement automaton accepts every trace that does not contain the sequence !ckLnAt?ckLnAt! InAt No? InAtNo.

Representing liveness properties. To describe a liveness property, we enclose an SD $S$ within an assert operator to indicate that the scenario represented by $S$ is the only valid 


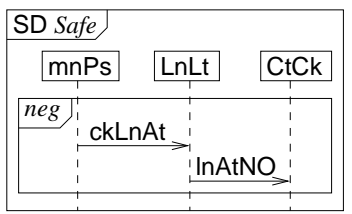

(a)

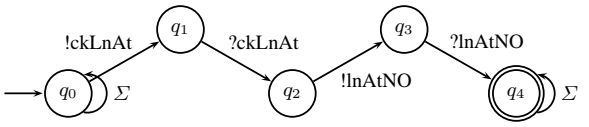

(b)

Fig. 6: (a) A Safe SD describing $P_{1}$ in Table 1 and (b) its corresponding NFA (before applying negate)

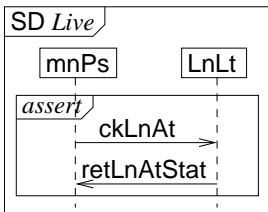

(a)

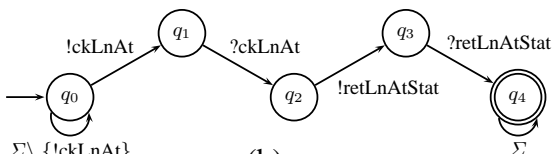

(b)

Fig. 7: (a) A Live SD describing $P_{2}$ in Table 1, and (b) its corresponding NFA

continuation of any system behavior [13]. For example, the SD in Fig. 7(a) describes the liveness property $P_{2}$ in Table 1 . After deriving the NFA $A_{S}$ for SD $S$, the automaton for assert $S$ is obtained by adding a self-loop to its initial state, allowing $A_{S}$ to wait for a satisfying run to begin. Intuitively, $A_{S}$ is a liveness automaton if every trace recognized by it includes the live part completely. Fig. 7(b) shows the automaton corresponding to the SD in Fig. 7(a). The resulting automaton accepts every trace that contains the entire sequence !ckLnAt?ckLnAt!ret LnAt Stat?retLnAt Stat.

Complexity of the Translation. The size of an automaton $A_{S}$ corresponding to a basic sequence diagram $S$ is $O\left(n^{k}\right)$ where $n$ is the number of events and $k$ is the number of processes [15]. Applying the sequence diagram operators does not cause a significant increase in the size of the resulting automata except for the negate operator that involves a determinzation step which can be exponential in the number of states of $A_{S}$. However, we note that in practice, the automata we have generated are relatively small, less than 9 states and 30 transitions [12]. Obviously, it remains to be seen whether the approach remains feasible for larger web service systems and more complex properties.

\section{SD Templates for Temporal Logic Property Patterns}

In this section, we introduce several templates expressed in the SD language for describing temporal logic property patterns [1]. We first provide an overview of these patterns in Section 4.1. We then describe our templates in the SD language in Section 4.2 and show how they can encode the property patterns.

\subsection{Temporal Logic Property Patterns}

The Specification Pattern System (SPS), proposed by Dwyer et al. [18], is a patternbased approach to the presentation, codification, and reuse of property specifications. The system allows patterns like "event $P$ is absent between events $Q$ and $S$ " or " $S$ precedes $P$ between $Q$ and $R$ " to be easily expressed in and translated between linear-time temporal logic (LTL) [19], computational tree logic (CTL) [19] and other state-based and event-based formalisms. SPS has been advocated as a standard tool for measuring 


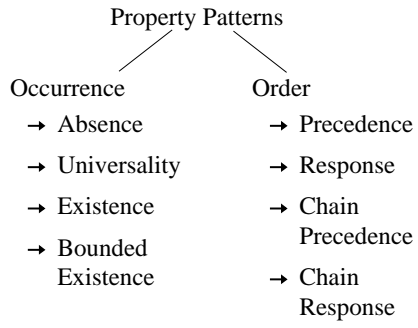

Fig. 8: Pattern Hierarchy.

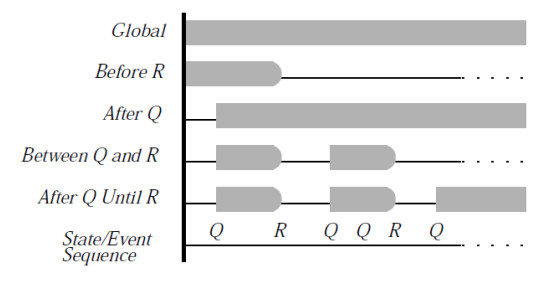

Fig. 9: Pattern Scopes.

the practical usefulness and expressive power of specification languages, e.g., [14] and [20].

The property patterns are organized into a hierarchy based on the kinds of system behaviors they describe (see Fig. 8): Occurrence patterns talk about the occurrence of a given event/state during system execution, and Order patterns specify relative order in which multiple events/states occur during system execution. The patterns are described below in detail:
Absence
An event does not occur within a given scope;
Existence
An event must occur within a given scope;
Bounded Existence An event can occur at most a certain number of times within a given scope;
Universality
An event must occur throughout a given scope;
Response An event must always be followed by another within a scope;
Response Chain A chain of events must always be followed by another chain of events within a scope;
Precedence An event must always be preceded by another within a scope;
Precedence Chain A chain of events must always be preceded by another chain of events within a scope.

Each pattern is associated with scopes - the regions of interest over which the pattern must hold. There are five basic kinds of scopes (depicted in Fig. 9):

\section{Global \\ Before $R$ \\ After $Q$ \\ Between $Q$ and $R$ After $Q$ until $R$}

The entire program execution;

The execution up to event $R$; The execution after event $Q$;

All parts of the execution between events $Q$ and $R$;

Similar to between, except that the designated part of the execution continues even if the second event does not occur.

For example, consider a property that says between every enqueue and empty messages, there must be a dequeue message. This property falls into the "Existence" pattern group because it indicates the occurrence of an event within a scope. The scope of this property is that of "Between" shown in Fig 9. Using the property pattern catalogue, the LTL formalization of the above property is as follows: $\square(($ enqueue $\wedge \neg$ empty $) \Rightarrow$ $(\neg$ empty $W($ dequeue $\wedge \neg$ empty $)))$.

\subsection{Mapping Property Patterns to SDs}

In this section, we provide several SD templates for the SPS patterns (see Fig 11), and show how these templates are mapped to the SPS pattern hierarchy. Selected mappings are described below; the remainder can be found in Appendix A. 


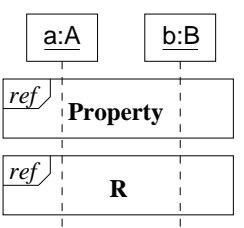

(a)

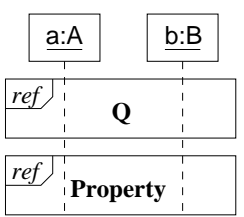

(b)

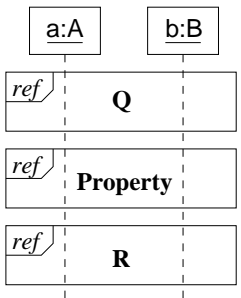

(c)

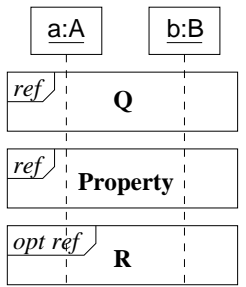

(d)

Fig. 10: Scope mapping for sequence diagrams: (a) Before $R$; (b) After $Q$; (c) Between $Q$ and $R$; and (d) After $Q$ until $R$.

Absence: message $\mathrm{p}$ cannot occur in a given scope. This can be expressed using a simple Safe SD, as shown in Fig. 11(a).

Existence: message $p$ must occur in a given scope. This can be expressed using a simple Live SD, as shown in Fig. 11(b).

Until: a sequence $\mathrm{p}^{*}$ of messages occurs until the first occurrence of message $\mathrm{q}$, in a given scope (see Fig. 11 (h)). This pattern is not part of the SPS; however, it is used to specify the precedence patterns. To express this pattern in the SD language, we note that this pattern, which can be formalized using a single until property [19], can be refuted in two ways only: a) p never occurs, or b) after seeing a finite number of $\mathrm{p}$ messages (expressed using loop 1, $n$ in Fig 11(h)), neither a p nor a q message occurs (expressed as $\neg\{\mathrm{p}, \mathrm{q}\}$ in Fig 11(h)).

Precedence: message $s$ (cause) precedes message $\mathrm{p}$ (effect), as shown in Fig. 11 (i). Note that this pattern allows the cause part to occur without the effect part. We describe this pattern in SD by expressing the two possible cases that this pattern specifies: a) p never occurs, or b) p never occurs before $s$. The first case corresponds to checking absence of $\mathrm{p}$; the second - to checking $\neg \mathrm{p} U \mathrm{~s}$, since we want to be sure that no $\mathrm{p}$ messages are sent before the first $\mathrm{s}$ message.

In the SDs in Fig. 11, symbols p, q, s, and t can denote more complex SDs, not just individual messages. In this case, we treat these symbols as place holders and use a ref operator to the SDs that should be inserted in place of these symbols. Also, in these cases, message complementation is replaced by negation. In Section 4.4, we provide detailed examples of how these patterns are used to specify properties of the LA system.

\subsection{Mapping Property Scopes}

We now show how to express property patterns involving scopes. Scopes are used to define the traces over which a property will be monitored. Scopes can be simple messages or more complex scenarios in our specification language. The ref operator is used to introduce scope delimiters in the corresponding locations. For example, to apply the Before $R$ scope to a property, the scope delimiter $R$ is inserted after the property we wish to verify (see Fig. 10(a)). In the case of After $Q$ scope, the delimiter is inserted before the property (see Fig. 10(b)). Finally, both the Between (see Fig. 10(c)) and After-until (see Fig. 10(d)) scopes add before/after delimiters. In the After-until scope, the property is valid even if the until part does not occur. Therefore, the second delimiter in this scope is optional.

\subsection{Specifying Properties of the Loan Application}

The following examples show how property patterns can be used to specify example properties of the LA system given in Table 1 . Properties $P_{1}$ and $P_{2}$ in that table corre- 


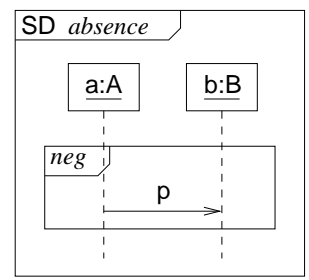

(a) Absence

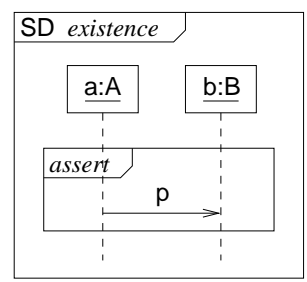

(b) Existence

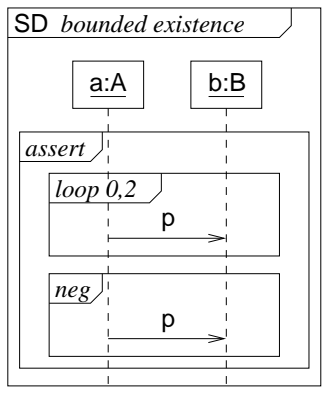

(c) Bounded Existence

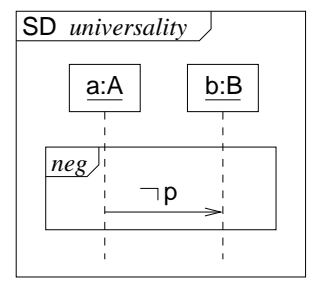

(d) Universality

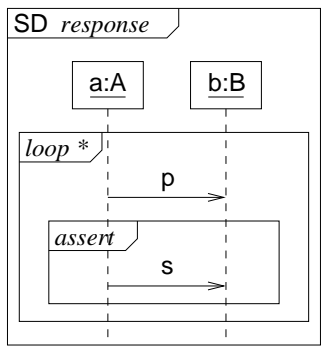

(e) Response

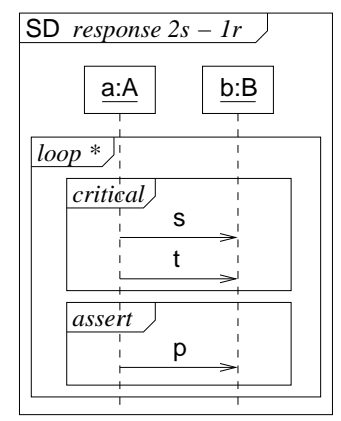

(f) Response Chain

2 stimulus - 1 response

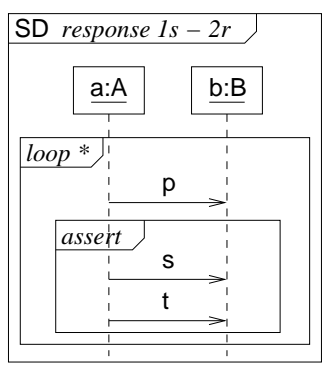

(g) Response Chain 1 stimulus - 2 response

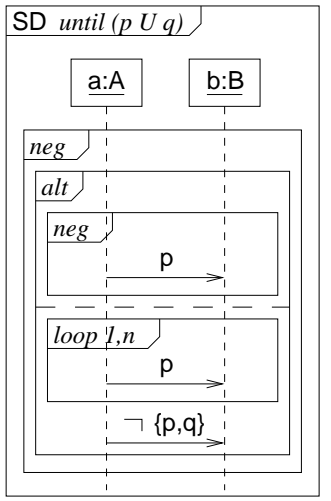

(h) Until

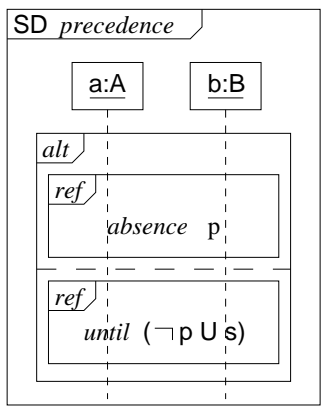

(i) Precedence

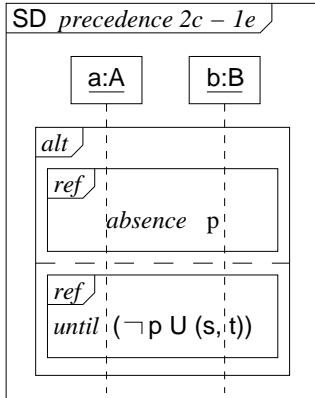

(j) Precedence Chain 2 cause - 1 effect

Fig. 11: Property pattern mapping for sequence diagrams 
spond to simple safe and live SDs and are described in Figs 6 and 7, respectively. The rest of the properties in that table are discussed below.

Property $P_{3}$ : A loan is considered granted if the outcome is either Complete TheLoan (CeLn), or ProcessTheApplication (PsAn). We express this property using the absence pattern (see Fig 11(a)): our property holds if there are no traces where a loan is granted after object LoanLimit (LnLt) warns that the loan amount is not okay. See Fig. 12 (a) for the corresponding SD; the monitor is shown in Fig. 12 (e). The remaining automata in Fig. 12 show intermediate steps in the contruction of the monitor.

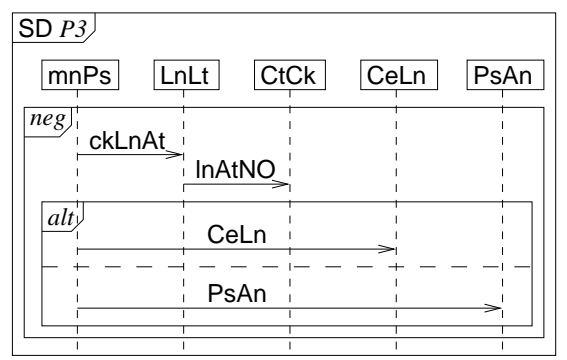

(a)

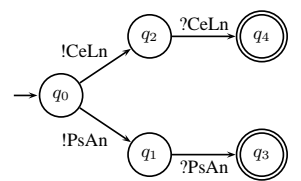

(b)

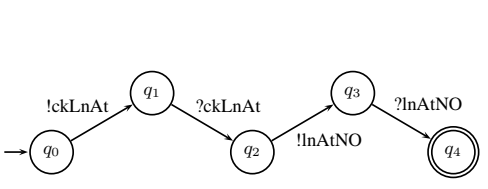

(c)

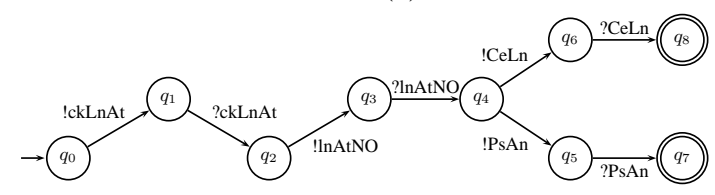

(d)

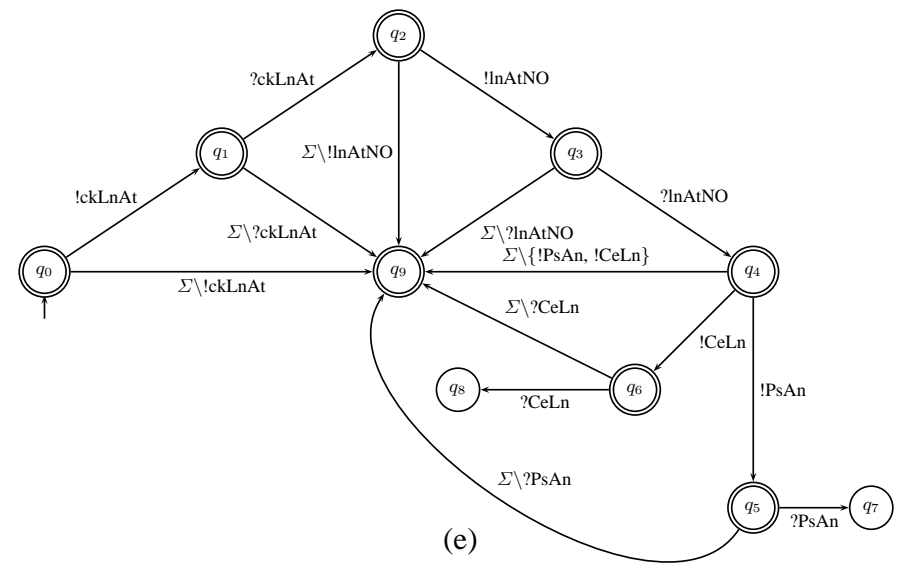

Fig. 12: $P_{3}$ : Absence pattern. (a) SD describing the LA property $P_{3}$ and its corresponding NFAs: (b) NFA for alt operator; (c) NFA for the first two messages; (d) NFA for first two messages, followed by the alt operator; (e) the resulting monitor (after applying negate).

Property $P_{4}$ : This property is a scoped version of $P_{3}$, i.e., $P_{4}$ is equivalent to the property $P_{3}$ scoped by the expression After $Q$ where $Q$ is "checking for a good credit score". So we only need to monitor the property $P_{3}$ on traces where a good credit score is already detected. To do this, we introduce the scope delimiter $Q$ before the 


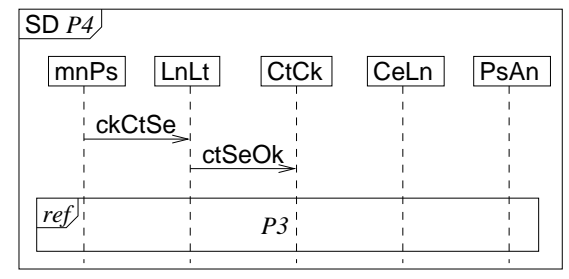

(a)

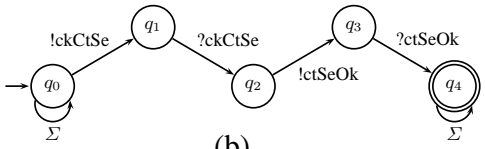

(b)

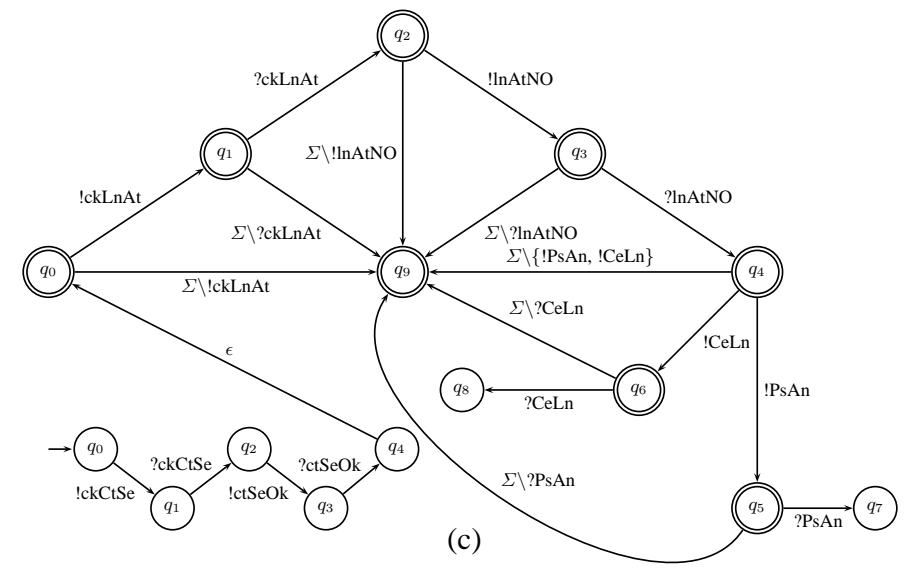

Fig. 13: $P_{4}$ : Scoped absence pattern. (a) SD describing the LA property $P_{4}$ and its corresponding NFAs: (b) NFA for scope $Q$; (c) the resulting monitor, obtained by concatenating the NFAs for the scope and $P_{3}$.

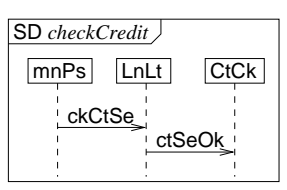

(a)

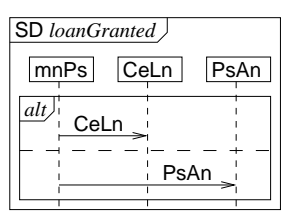

(b)

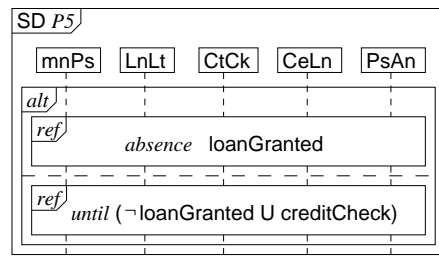

(c)

Fig. 14: $P_{5}$ : Precedence pattern. (a) SD for checkCredit; (b) SD for loanGranted; (c) SD showing application of precedence pattern.

property $P_{3}$, as seen in Fig 10(b). The SD corresponding to $P_{4}$ is shown in Fig 13(a) and consists of two parts: (1) scope $Q$ and (2) property $P_{3}$, i.e., the fragment specified by a ref operator which should be replaced by the SD for $P_{3}$. The monitor for the whole property $P_{4}$ is shown in Fig. 13(b).

Property $P_{5}$ : We can express property $P_{5}$ using the precedence pattern by indicating that the scenario creditCheck must precede the scenario loanGranted. Note that in this pattern, creditCheck is not optional and it must occur for the property to hold. Figs. 14 (a) and (b) show the SDs for creditCheck and loanGranted, respectively. The SD for $P_{5}$ is shown in Fig. 14 (c) which is an instantiation of the Precedence pattern in Fig 11(i) where $\mathrm{p}$ is replaced by loanGranted, and $\mathrm{s}-$ by creditCheck. In this pattern, message $\mathrm{p}$ is replaced by a scenario, and hence, scenario negation is used instead of message complementation. 


\section{Experience and Tool Support}

We have implemented our runtime monitoring framework within the IBMWebSphere ${ }^{\circledR}$ business integration products [21]. In what follows, we describe the architecture of our solution and its intended use. We also report on preliminary experience of using this framework to check correctness of web services. For implementation details, see [12].

Our solution uses the WebSphere Process Server (WPS) [22] and the WebSphere Integration Developer (WID) [23]. The former provides a BPEL-compliant process engine for executing BPEL processes and a built-in Service Component Architecture (SCA), which is a particular instantiation of SOA. The latter provides a development environment for building web service applications and a graphical package for creating UML Sequence Diagrams.

During and after application development, users can create UML SD specifications for their web service applications within the WID environment. If monitoring is enabled, our framework translates these diagrams into monitor automata using the techniques in Section 3. During the execution of the web service, interaction events from the WPS are sent to our framework. These events are used to update the state of every active monitor automaton, until an error has been found or all partners terminate.

Our patterns are available as editable UML sequence diagrams (. dnx files). Users must first add these files to the WID project of the application they wish to monitor. These patterns can now be modified to create actual system properties, using our Sequence Diagram editor.

We applied our framework to the Loan Application system, with the goal of specifying and checking the properties mentioned in Table 1. On normal execution traces of this system, these properties should never fail, as this application implements the workflow shown in Fig 1(a). As it is a sample application, some details have been simplified. For example, the CreditCheck component generates random credit scores.

Testing the system, we realized that properties $P_{1}, P_{3}$, and $P_{4}$ fail on a trace in which taxpayer id is 1888 and loan amount is -1000 . This execution trace is as follows: ckCtSe, ctSeOK, ckLnAt InAtNo, CeLn. It can be seen that: (1) $P_{1}$ fails because of event InAtNo that indicates that the loan amount is not greater than zero; (2) $P_{3}$ is violated because a forbidden behavior ckLnAt, InAtNO,CeLn, meaning that an invalid loan is accepted, appears in the above trace; (3) $P_{4}$ fails for the same reason that $P_{3}$ fails because $P_{4}$ is a scoped version of $P_{3}$. Note that properties $P_{2}$ and $P_{5}$ in Table 1 are not violated by the above trace.

After examining the LA system source code, we realized that the application was distributed with some hard-coded logic, presumably to facilitate testing purposes. An applicant with a taxpayer id that ends with " 888 " is always trusted and given a good credit score (instead of a random score). Since the loan amount is less than $\$ 50,001$, this loan is automatically approved because of the good credit score assigned. We also realized that the application is not validating its input.

To report monitoring results, we display the cause of the violations in the Sequence Diagram editor. This will either be due to the occurrence of an unexpected event, or the existence of an incomplete sequence. Unexpected events are highlighted in the corresponding SDs (see Fig 15 (a)). In the case of incomplete sequences, we mark the termination location (see Fig 15 (b)). 


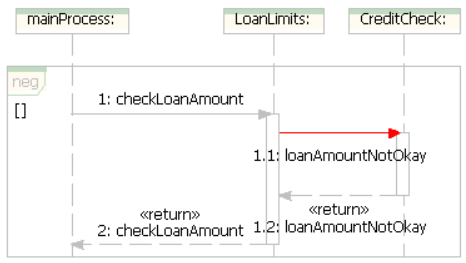

(a)

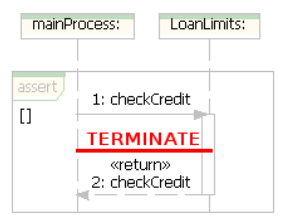

(b)

Fig. 15: Reporting errors: (a) unexpected event, (b) incomplete sequence

\section{Conclusion}

In this paper, we described our framework for runtime monitoring of web service conversations developed as part of an industrial-strength system. The framework is an aggregation of existing runtime verification techniques and is a continuation of [12]. It is non-intrusive, running in parallel with the monitored system and intercepting interaction events during run time. Thus, it does not require any code instrumentation, does not significantly affect the performance of the monitored system, and enables reasoning about partners expressed in different languages. Furthermore, the use of a subset of UML 2.0 SDs as a specification language ensures that the framework is usable by practitioners to specify a wide range of properties. By formalizing this subset using automata, we can check finite execution traces of web services against these properties. Liveness becomes finitary, where user-specified time limits or the process termination act as the stopping conditions.

We have successfully mapped all the Specification Property System patterns into our SD subset. The availability of customizable patterns should improve the usability of our specification language. More complex conversations can be checked, as it is easy to build properties through SD composition. Using SD references, our properties are also easier to read, since details can be hidden. Finally, we have created a library of such sequence diagram patterns and showed how patterns can be used to specify monitors which lead to discovery of bugs in real webservice applications.

\section{Related Work.}

Like other partial-order scenario-based formalisms such as MSCs [24] and LSCs [25], UML 2.0 Sequence Diagrams are enjoying an increasing usage as specification languages. [14] proposes a Property Sequence Chart (PSC) language, which is an extended notation of a subset of UML 2.0 SDs. PSC enables expressing safety and liveness properties by assigning attributes fail and required to messages. This is equivalent to applying operators negate and assert to individual SD message, respectively. The semantics of PSC is given using Büchi Automata, designed to operate on infinite execution traces. Since we consider only finite executions of web services, automata over finite words are sufficient and significantly easier to implement. Finally, in [26], Stahl maps BPEL schemas into Petri nets and utilises a verification tool LORA (Low Level Petri net Analyzer) to verify CTL properties.

Future Work. While the initial experience using the framework has been positive, we need to address a number of issues before it becomes fully usable. The first set of issues deals with increasing the range of properties that can be specified and monitored. In the examples presented here, all objects were unique, whereas in practice, users may be interested in verifying interactions between multiple processes of the same type. For example, a user with a good credit score may concurrently apply for two loans, each 
for less than $\$ 50001$, to bypass the manual approval required for a loan for the total amount. In this case, two bank branches may want to communicate to avoid this kind of situation. We feel that the problem can be easily solved by encoding process IDs into the specification, the automata transition relation, and interaction events.

We also plan to begin investigation of techniques to help locate cause of errors from seeing results of successful and unsuccessful runs of the system. For example, given a monitor violation, we would like to produce similar conversations that do not cause a violation, so as to help pinpoint cause of the violation (as the place signaled with the violation is not necessarily the cause). We will experiment with the techniques in [27, 28] for this task.

On a side note, our work so far has been built on a basis that all partners operate within the same process server and thus a centralized monitor is a viable option. In practice, most web services are distributed, requiring a distributed monitoring framework. We plan to investigate techniques used in the DESERT project [29] to turn a centralized monitor into a set of distributed ones, running in different process servers.

\section{References}

1. Dwyer, M., Avrunin, G., Corbett, J.: "Patterns in Property Specifications for Finite-State Verification". In: Proceedings of 21st International Conference on Software Engineering (ICSE’99). (May 1999) 411-420

2. IBM: Business Process Execution Language for Web Services. http://www-128.ibm.com/ developerworks/library/specification\%/ws-bpel/

3. Fu, X., Bultan, T., Su, J.: "Conversation Protocols: A Formalism for Specification and Verification of Reactive Electronic Services.". In: In Proceedings of the Eighth International Conference on Implementation and Application of Automata (CIAA 2003), Santa Barbara, California (July 2003) 188-200

4. Fu, X., Bultan, T., Su, J.: “Analysis of Interacting BPEL Web Services.”. In: Proceedings of the Thirteenth International World Wide Web Conference (WWW 2004), New York, NY (May 2004) 621-630

5. Kazhamiakin, R., Pistore, M.: "A Parametric Communication Model for the Verification of BPEL4WS Compositions”. In: EPEW/WS-FM. (2005) 318-332

6. Baldoni, M., Baroglio, C., Martelli, A., Patti, V., Schifanella, C.: "Verifying the Conformance of Web Services to Global Interaction Protocols: A First Step”. In: EPEW/WS-FM. (2005) 257-271

7. Foster, H., Uchitel, S., Magee, J., Kramer, J.: "Model-based Verification of Web Service Compositions". In: Proceedings of 18th IEEE International Conference on Automated Software Engineering (ASE 2003), IEEE Computer Society (2003) 152-163

8. Ghafari, N., Gurfinkel, A., Klarlund, N., Trefler, R.: "Algorithmic Analysis of Piecewise FIFO Systems". In: Proceedings of 7th International Conference on Formal Methods in Computer-Aided Design (FMCAD’07). LNCS, Austin, Texas (November 2007) 45-52

9. Baresi, L., Ghezzi, C., Guinea, S.: “Smart Monitors for Composed Services”. In: ICSOC'04. (2004) 193-202

10. Robinson, W.N.: “Monitoring Web Service Requirements". In: Proceedings of RE'03. (2003) 65-74

11. Mahbub, K., Spanoudakis, G.: "Run-time Monitoring of Requirements for Systems Composed of Web-Services: Initial Implementation and Evaluation Experience". In: Proceedings of ICWS'05. (2005) 257-265

12. Gan, Y., Chechik, M., Nejati, S., Bennett, J., O'Farrell, B., Waterhouse, J.: "Runtime Monitoring of Web Service Conversations”. In: Proceedings of CASCON'07. (November 2007) 
13. Object Management Group (OMG): Unified Modeling Language (UML 2.0). http: // www. uml.org/

14. Autili, M., Inverardi, P., Pelliccione, P.: "A Scenario Based Notation for Specifying Temporal Properties”. In: Proceedings of SCESM, ICSE'06 Workshop. (2006)

15. Alur, R., Yannakakis, M.: "Model Checking of Message Sequence Charts". In: Proceedings of CONCUR'99. (1999) 114-129

16. Grosu, R., Smolka, S.A.: "Safety-Liveness Semantics for UML 2.0 Sequence Diagrams". In: ACSD'05. (2005) 6-14

17. Hopcroft, J.E., Ullman, J.D.: Introduction to Automata Theory, Languages and Computation. Addison Wesley (1979)

18. Dwyer, M.B., Avrunin, G.S., Corbett, J.C.: "Property Specification Patterns for Finite-state Verification". In: Proceedings of 2nd Workshop on Formal Methods in Software Practice. (March 1998)

19. Clarke, E., Grumberg, O., Peled, D.: Model Checking. MIT Press (1999)

20. Yu, J., Manh, T.P., Han, J., Jin, Y., Han, Y., Wang, J.: Pattern Based Property Specification and Verification for Service Composition. In: Proceedings of 7th International Conference on Web Information Systems Engineering (WISE 2006). (2006) 156-168

21. IBM: WebSphere Business Integration Software. http://www-306.ibm.com/software/ infol/websphere/index. jsp?t $\%$ ab=products/businessint

22. IBM: WebSphere Process Server. http://www-306.ibm.com/software/integration/wps/

23. IBM: WebSphere Integration Developer. http://www-306.ibm.com/software/ integration/wid/

24. ITU-TS: "ITU-TS Recommendation Z.120: Message Sequence Chart 1996 (MSC96)". Technical report, ITU-TS, Geneva (1996)

25. Damm, W., Harel, D.: "LSCs: Breathing Life into Message Sequence Charts.". Formal Methods in System Design 19(1) (2001) 45-80

26. Stahl, C.: "A Petri Net Semantics for BPEL". Technical Report 188, Humboldt-Universität zu Berlin, Institut für Informatik (2005)

27. Zeller, A.: Isolating cause-effect chains from computer programs. SIGSOFT Softw. Eng. Notes 27(6) (2002) 1-10

28. Groce, A., Chaki, S., Kroening, D., Strichman, O.: Error explanation with distance metrics. Int. J. Softw. Tools Technol. Transf. 8(3) (2006) 229-247

29. Inverardi, P., Mostarda, L., Tivoli, M., Autili, M.: "Synthesis of Correct and Distributed Adaptors for Component-Based Systems: an Automatic Approach". In: Proceedings of ASE'05. (2005) 405-409 


\section{A Other Property Patterns}

$k$-Bounded Existence: message p can occur at most $k$ times in a given scope. We can check the existence of at most $k$ messages using the loop operator. After the loop, we need to check that $\mathrm{p}$ does not occur, which corresponds to the absence pattern (see Fig. 11 (c)).

Universality: only a sequence $\mathrm{p}^{*}$ of messages can occur in a given scope. This is equivalent to checking for the absence of complement messages (see Fig. 11 (d)).

Response: message $\mathrm{p}$ (stimulus) must be followed by message $\mathrm{s}$ (response), in a given scope. A response can occur without stimuli, so the stimulus is represented using a regular message, whereas the response is mandatory. The existence of stimulus/response pairs are checked in an infinite loop, as there can be many stimulus/response pairs in one execution trace (see Fig. 11 (e)).

Response Chain: a sequence $\mathrm{p}_{1}, \ldots, \mathrm{p}_{n}$ of messages must be followed by the sequence $q_{1}, \ldots, q_{m}$ of messages, in a given scope. We show two examples of this pattern: $p$ responds to $s, t$ (see Fig. 11 (f)), and $s$, t responds to $p$ (see Fig. 11 (g)). Response chain patterns have the same basic form of the response pattern.

- $p$ responds to $s, t: 2$ stimulus -1 response. The critical operator is used to enclose the message sequence $s, t$, to ensure atomicity of this sequence. An assert cannot be used since the stimulus sequence is optional.

- $s, t$ responds to $p: 1$ stimulus -2 response. The message sequence now occurs within the assert operator, so an additional critical operator would be superfluous).

Precedence Chain: a sequence $\mathrm{p}_{1}, \ldots, \mathrm{p}_{n}$ of messages must precede the sequence $\mathrm{q}_{1}, \ldots, \mathrm{q}_{m}$ of messages, in a given scope. We show an example of this pattern, 2 cause -1 effect, $p$ is preceded by $s, t$ (see Fig. $11(j)$ ). This pattern is mapped using the absence and until patterns, just like in the precedence pattern. The implicit negate operators in the absence and until patterns handle the message sequences, so there is no need to add critical operators. 


\section{B Framework}

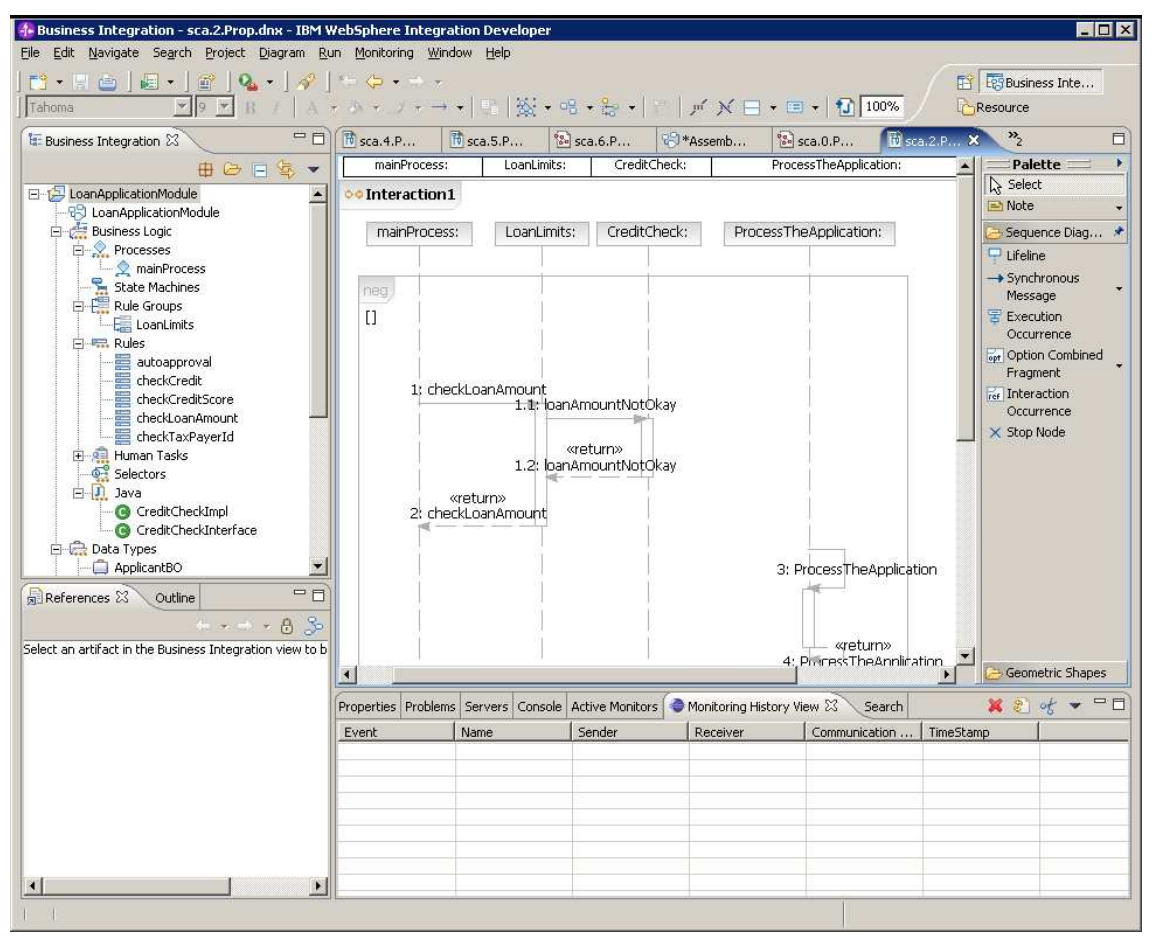

Fig. 16: Screenshot of the Framework's User Interface. 